MR. FÉLIX PHAM (Orcid ID : 0000-0002-7855-4930)

DR CATHERINE SCHISSLER (Orcid ID : 0000-0002-0340-0988)

PROFESSOR D LIPSKER (Orcid ID : 0000-0003-4675-0336)

Article type : Correspondence

\title{
Diagnostic value of skin biopsy in autoinflammatory diseases for patients with recurrent fever and urticarial eruption
}

Dear Editor,

Recurrent fever is defined by two episodes of fever separated by a free interval of at least two weeks. ${ }^{1}$ Diseases causing recurrent fever may be classified into three categories: infections, neoplasia, and non-infectious inflammations. Recurrent fever may be associated with autoinflammatory diseases like Schnitzler syndrome, NLRP3-associated autoinflammatory diseases (NLRP3-AID, formerly cryopyrin associated periodic syndrome or CAPS), or adult onset Still's disease (AOSD). ${ }^{2}$ Those diseases are characterized by an urticarial eruption with pale pink macules and slightly elevated papules and plaques lasting 24 to 48 hours. ${ }^{3}$ Histopathology typically shows a neutrophilic urticarial dermatosis (NUD), a dense perivascular and interstitial neutrophilic infiltrate with leukocytoclasia but without vasculitis or dermal oedema. ${ }^{4,5}$

The goal of this study was to assess the diagnostic value of skin biopsy for the diagnosis of autoinflammatory diseases like Schnitzler syndrome in patients with recurrent fever and urticarial eruption. Such patients were consecutively examined at the National Amyloidosis Centre (NAC) in London between October 2012 and July 2015. Clinical, biological, and genetic data were retrieved by the senior physician of the NAC who followed them (HL). Their skin biopsies were reviewed in the Histopathology Laboratory of the Strasbourg University Hospitals by a

This article has been accepted for publication and undergone full peer review but has not been through the copyediting, typesetting, pagination and proofreading process, which may lead to differences between this version and the Version of Record. Please cite this article as doi: 10.1111/CED. 14508

This article is protected by copyright. All rights reserved 
dermatopathologist (DL) unaware of the final diagnosis. He suggested a diagnosis based solely on histopathological data. Subsequently, biopsies could be grouped into two categories: neutrophilic disease (ND) or non-neutrophilic disease (non-ND). The final diagnosis established by HL was subsequently collected.

We calculated sensitivity, specificity, positive predictive value (PPV) and negative predictive value (NPV) of the histological aspect for the diagnosis of Schnitzler syndrome, NLRP3-AID, Schnitzler syndrome in combination with NLRP3-AID and of "non-Schnitzler, non-NLRP3-AID" and the associated confidence interval (CI) at 95\%. All the inferential analyses were performed using Bayesian methods.

Twenty biopsies from twenty patients were studied. One biopsy of seborrheic keratosis was excluded. We studied 19 cases that met the inclusion criteria. Every patient had a recurrent fever and an urticarial eruption, clinically compatible with NUD. Table 1 summarizes all the clinical, biological and histological data, the final diagnosis as well as the disease course.

Nine patients had a ND with an aspect suggestive of NUD in five, because of an interstitial infiltrate of neutrophils with leukocytoclasia throughout the dermis without vasculitis and without oedema.

Ten patients had non-ND: four had histopathology compatible with common urticaria; one had a very deep monocyte infiltration spreading into the deep hypodermis, highly suggestive of TNF receptor associated periodic syndrome (TRAPS); one "neutrophilic urticaria" corresponded to the final diagnosis of urticarial vasculitis as repeated biopsies revealed leukocytoclastic vasculitis; four had an unspecific aspect.

Altogether, there were eleven patients with autoinflammatory diseases: four Schnitzler syndromes, two "variants" of Schnitzler syndrome (one without any gammopathy and one with IgG-variant), four NLRP3-AID and one TRAPS. Two patients had urticarial vasculitis, one delayed pressure urticaria, one chronic spontaneous urticaria and four indefinite diagnoses.

ND had a sensitivity, specificity, PPV and NPV of 0.83 (95\% CI 0.48-0.99), 0.65 (95\% CI 0.410.85 ), 0.46 (95\% CI 0.19-0.74) and 0.92 (95\% CI 0.71-1), respectively, for the diagnosis of IgMSchnitzler syndrome. Especially, NUD had a specificity and a sensitivity of 0.91 (95\% CI 0.69-1) and 0.50 (95\% CI 0.21-0.79), respectively, for the diagnosis of NLRP3-AID or Schnitzler syndrome. 
This study of a consecutive case series of 19 patients with recurrent fever and urticarial eruption, examined at the NAC, confirms that a histopathological appearance of ND is a finding with a high sensitivity for the diagnosis of IgM-Schnitzler syndrome as every patient matching the diagnostic criteria had a neutrophilic infiltrate in the biopsy. ${ }^{6} \mathrm{NPV}$ was excellent, so that Schnitzler syndrome becomes unlikely in the absence of a neutrophilic infiltrate in this situation.

Histopathological aspect of NUD is mainly associated with Schnitzler syndrome, NLRP3-AID, AOSD and systemic lupus erythematosus. ${ }^{4,7}$ NLRP3-AID are characterized histologically by a neutrophilic infiltrate similar to a NUD with distinctive perieccrine infiltration of neutrophils. ${ }^{8}$ The main limitations of this study were the limited number of patients, which explains the broad CI limits, and the fact that NAC is a tertiary and reference centre for amyloidosis and autoinflammatory diseases in England: patients examined herein have an important number of complementary exams and common diseases are usually ruled out before referral. This limits the generalizability of the diagnostic values of skin biopsy presented herein.

Histopathological aspect of ND in patients with recurrent fever and urticarial eruption should bring the diagnosis of NLRP3-AID and Schnitzler syndrome to mind. Nevertheless, the absence of ND cannot definitively exclude those diagnosis, but renders at least the diagnosis of Schnitzler syndrome unlikely.

F. Pham, ${ }^{1}$ C. Schissler, ${ }^{1}$ M. Schaeffer ${ }^{2}$ H.-J. Lachmann ${ }^{3}$ and D. Lipsker ${ }^{1,4}$

${ }^{1}$ Dermatology Clinic, Strasbourg University Hospitals, Strasbourg, France

${ }^{2}$ Clinical Research Methodology Group, Public Health Service, Strasbourg University Hospitals, Strasbourg, France

${ }^{3}$ National Amyloidosis Centre, University College London, Gower Street, London, UnitedKingdom

${ }^{4}$ Faculty of Medicine, University of Strasbourg, Strasbourg, France

Correspondence: Dan Lipsker

Email: dan.lipsker@chru-strasbourg.fr 


\section{Funding sources: none.}

Conflicts of interest: none to declare.

\section{References}

1Knockaert DC. Recurrent Fevers of Unknown Origin. Infectious Disease Clinics of North America 2007; 21:1189-211.

2Kallinich $\mathrm{T}$, Gattorno $\mathrm{M}$, Grattan CE, et al. Unexplained recurrent fever: when is autoinflammation the explanation? Allergy 2013; 68:285-96.

3 Kacar M, Pathak S, Savic S. Hereditary systemic autoinflammatory diseases and Schnitzler's syndrome. Rheumatology 2019; 58:vi31-43.

4Kieffer C, Cribier B, Lipsker D. Neutrophilic Urticarial Dermatosis: A Variant of Neutrophilic Urticaria Strongly Associated With Systemic Disease. Report of 9 New Cases and Review of the Literature. Medicine 2009; 88:23-31.

5Broekaert SMC, Böer-Auer A, Kerl K, et al. Neutrophilic Epitheliotropism Is a Histopathological Clue to Neutrophilic Urticarial Dermatosis: The American Journal of Dermatopathology 2016; 38:39-49.

6Lipsker D, Veran Y, Grunenberger F, et al. The Schnitzler Syndrome: Four New Cases and Review of the Literature. Medicine 2001; 80:37-44.

7 Gusdorf L, Bessis D, Lipsker D. Lupus Erythematosus and Neutrophilic Urticarial Dermatosis: A Retrospective Study of 7 Patients. Medicine 2014; 93:e351.

8Kolivras A, Theunis A, Ferster A, et al. Cryopyrin-associated periodic syndrome: an autoinflammatory disease manifested as neutrophilic urticarial dermatosis with additional perieccrine involvement. Journal of Cutaneous Pathology 2011; 38:202-8. 


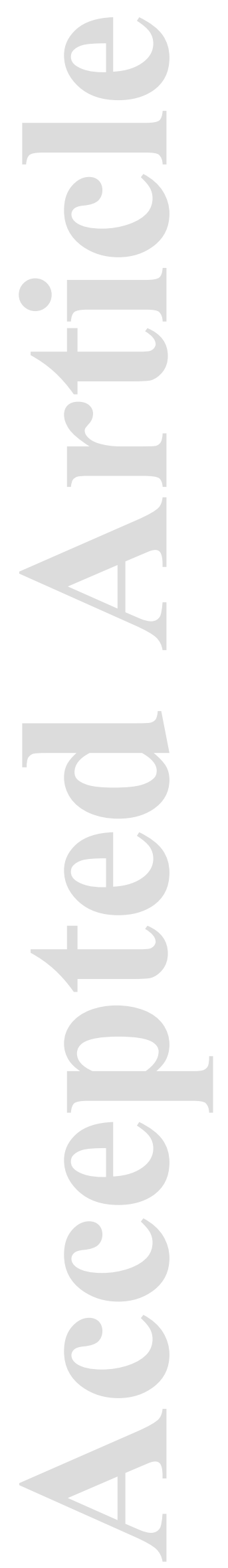

This article is protected by copyright. All rights reserved 
Table 1. Demographic, biologic, genetic, diagnostic data and disease course under anti-IL1 antibody of patients with recurrent fever and urticarial eruption

\begin{tabular}{|c|c|c|c|c|c|c|c|c|}
\hline ) & $\begin{array}{l}\text { Age/ } \\
\text { Sex }\end{array}$ & $\begin{array}{l}\mathrm{DD} \\
\text { (y) }\end{array}$ & $\begin{array}{c}\text { CRP } \\
(\mathrm{mg} / \mathrm{L})\end{array}$ & $\begin{array}{l}\text { Monoclonal } \\
\text { gammopathy }\end{array}$ & Histology & Genetic & Final diagnosis & $\begin{array}{c}\text { Evolution, anti- } \\
\text { IL1 }\end{array}$ \\
\hline 1 & $68 / \mathrm{M}$ & 16 & 8 & IgM kappa & Neutrophilic urticarial dermatosis & NLRP3- & SchS & CR, anakinra \\
\hline 2 & $68 / \mathrm{M}$ & 9 & 79 & IgM lambda & Neutrophilic urticarial dermatosis & NLRP3- & SchS & $\mathrm{CR}$, anakinra \\
\hline 3 & $49 / \mathrm{M}$ & 12 & 40 & IgM kappa & Neutrophilic urticarial dermatosis & NLRP3- & SchS & $\mathrm{CR}$, anakinra \\
\hline 4 & $64 / \mathrm{M}$ & 11 & 45 & IgM lambda & Neutrophilic dermatosis & NLRP3- & SchS & $\mathrm{CR}$, anakinra \\
\hline 5 & $69 / \mathrm{M}$ & 10 & 6 & - & Neutrophilic urticarial dermatosis & NLRP3- & "variant" of SchS & $\mathrm{CR}$, anakinra \\
\hline 6 & $60 / \mathrm{M}$ & 4 & 18 & IgG kappa & Chronic urticaria & NLRP3- & "variant" of SchS & PR, anakinra \\
\hline 7 & $54 / \mathrm{M}$ & 14 & - & - & Neutrophilic urticarial dermatosis & mosaic, $N L R P 3+$ & NLRP3-AID & $\mathrm{CR}$, anakinra \\
\hline 8 & $32 / \mathrm{M}$ & 25 & - & - & Neutrophilic urticarial dermatosis & NLRP3+ & NLRP3-AID & $\mathrm{CR}$, canakinumab \\
\hline 9 & $24 / \mathrm{F}$ & 15 & Increased & - & Chronic urticaria & NLRP3+ & NLRP3-AID & CR, anakinra \\
\hline 10 & $62 / \mathrm{F}$ & 55 & N/A & - & Not specific & NLRP3+ & NLRP3-AID & $\mathrm{CR}$, anakinra \\
\hline 11 & $42 / \mathrm{M}$ & 15 & N/A & - & TRAPS & TNFRSF1A+ & TRAPS & $\mathrm{CR}$, anakinra \\
\hline 12 & $69 / \mathrm{F}$ & 9 & 11 & - & Spongiform dermatosis & NLRP3- NLRP12- TNFRSF1A- & Urticarial vasculitis & $\mathrm{N} / \mathrm{A}$ \\
\hline 13 & $19 / \mathrm{F}$ & N/A & N/A & - & Neutrophilic urticaria & N/A & Urticarial vasculitis & N/A \\
\hline
\end{tabular}




\begin{tabular}{|c|c|c|c|c|c|c|c|c|}
\hline 14 & $58 / \mathrm{F}$ & 45 & - & - & Neutrophilic dermatosis & NLRP3- NLRP12- TNFRSF1A- & Physical urticaria & N/A \\
\hline 15 & $46 / \mathrm{F}$ & 6 & - & - & Chronic urticaria & $\mathrm{N} / \mathrm{A}$ & Chronic urticaria & $\mathrm{N} / \mathrm{A}$ \\
\hline 16 & $71 / \mathrm{M}$ & N/A & 50 & - & Neutrophilic dermatosis & $M E F V-T N F R S F 1 A-N L R P 3-M V K-$ & $\mathrm{N} / \mathrm{D}$ & N/A \\
\hline 17 & 63/M & 30 & - & IgG kappa & Chronic urticaria & $M E F V-T N F R S F 1 A-N L R P 3-M V K-$ & $\mathrm{N} / \mathrm{D}$ & $\mathrm{NR}$, anakinra \\
\hline $18^{\S}$ & $50 / \mathrm{M}$ & N/A & - & IgG lambda & Psoriasiform and spongiform dermatosis & $M E F V-T N F R S F 1 A-N L R P 3-M V K-$ & $\mathrm{N} / \mathrm{D}$ & N/A \\
\hline 19 & $45 / \mathrm{F}$ & 3 & - & - & Spongiform dermatosis & MEFV-TNFRSF1A-NLRP3- MVK- & $\mathrm{N} / \mathrm{D}$ & N/A \\
\hline
\end{tabular}

F, female; M, male; DD, disease duration; y, years; N/A, not available; NLRP3-AID, NLRP3-associated inflammatory diseases; SchS, Schnitzler syndrome; N/D, no definite diagnosis; CR, complete response; NR, no response; PR, partial response; TRAPS, TNF receptor associated periodic syndrome; \$Positive antinuclear antibodies at 1:1280 (titer) 1 